\title{
Comparison of conventional transrectal ultrasound, magnetic resonance imaging, and micro-ultrasound for visualizing prostate cancer in an active surveillance population: A feasibility study
}

\author{
Gregg Eure ${ }^{1}$; Daryl Fanney²; Jefferson Lin ${ }^{1}$; Brian Wodlinger ${ }^{3}$; Sangeet Ghai ${ }^{4}$ \\ ${ }^{1}$ Urology of Virginia, Virginia Beach, VA, United States; ${ }^{2}$ MRI \& CT Diagnostics, Virginia Beach, VA, United \\ States; ${ }^{3}$ Exact Imaging, Markham, ON, Canada; ${ }^{4}$ Joint Department of Medical Imaging, University of Toronto, \\ University Health Network - Mount Sinai Hospital-Women's College Hospital, Toronto General Hospital, Toronto, \\ ON, Canada
}

Funding: This was a sponsored study with clinical costs covered by Exact Imaging.

Cite as: Can Urol Assoc J 2018 August 30; Epub ahead of print. http://dx.doi.org/10.5489/cuaj.5361

Published online August 30, 2018

$* * *$

\begin{abstract}
Introduction: Active surveillance monitoring of prostate cancer is unique in that most patients have low-grade disease that is not well-visualized by any common imaging technique. Highresolution (29 MHz) micro-ultrasound is a new, real-time modality that has been demonstrated to be sensitive to significant prostate cancer and effective for biopsy targeting. This study compares micro-ultrasound imaging with magnetic resonance imaging (MRI) and conventional ultrasound for visualizing prostate cancer in active surveillance.
\end{abstract}

Methods: Nine patients on active surveillance were imaged with multiparametric (mp) MRI prior to biopsy. During the biopsy procedure, imaging and target identification was first performed using conventional ultrasound, then using micro-ultrasound. The mpMRI report was then unblinded and used to determine cognitive fusion targets. Using micro-ultrasound, biopsy samples were taken from targets in each modality, plus 12 systematic samples.

Results: mpMRI and micro-ultrasound both demonstrated superior sensitivity to Gleason sum 7 or higher cancer compared to conventional ultrasound ( $\mathrm{p}=0.02 \mathrm{McNemar}$ 's test). Microultrasound detected $89 \%$ of clinically significant cancer, compared to 56\% for mpMRI.

Conclusions: Micro-ultrasound may provide similar sensitivity to clinically significant prostate cancer as mpMRI and visualize all significant mpMRI targets. Unlike mpMRI, micro-ultrasound is performed in the office, in real-time during the biopsy procedure, and so is expected to 
maintain the cost-effectiveness of conventional ultrasound. Larger studies are needed before these results may be applied in a clinical setting.

\section{Introduction}

Prostate cancer is the most common cancer in men in the western world, but only the third most common cause of cancer-related death in Europe and the fourth in America. ${ }^{1-3}$ This discrepancy is due to the diverse nature of the disease, with some cases proceeding aggressively and others remaining indolent for many years. Active surveillance protocols are intended for men with lowgrade prostate cancer to safely remain untreated yet under supervision until disease progression is detected. Unfortunately, conventional ultrasound-based transrectal systematic biopsy, the current standard of care to monitor prostate cancer, is insufficient and even with repeated prostate biopsy every 6 to 24 months, pathology results often differ significantly with $37-48 \%$ of men with known low-grade cancer receiving a benign diagnosis on confirmatory biopsy. ${ }^{4-6}$ This high false negative rate highlights the insensitivity of the systematic biopsy procedure, which likely results in men with aggressive disease being misclassified and incorrectly included in active surveillance protocols instead of receiving appropriate treatment. Similarly, this false negative rate may also cause delays in determining disease progression. Both of these results limit options for curative treatment and expose patients to a repeated invasive biopsy procedure with associated risks for morbidity.

Multi-parametric MRI (mpMRI) and high resolution micro-ultrasound have both been proposed as novel imaging techniques that may improve patient care. ${ }^{7-9}$ While mpMRI has been well researched and demonstrated to improve accuracy in identifying prostate cancer compared to conventional ultrasound, it has still been found to miss up to $23 \%$ (meta-analysis mean $12.2 \%$ )

of GS 7+ lesions. ${ }^{10,11}$ Additionally, it is highly dependent on operator expertise in both performing mpMRI as well as in reading and interpreting PI-RADS results, with one study reporting only 59\% agreement for PZ lesions even amongst expert readers. ${ }^{12}$ Prospective studies of the positive predictive value and sensitivity have also raised concerns about bias in many of the retrospective studies demonstrating very high accuracy. ${ }^{13}$ Other drawbacks to the utility of mpMRI include significant capital and operational costs, workflow complexity, reliance on expert radiological resources, patient inconvenience, and lack of freely available MRI access. It therefore cannot yet be recommended as a replacement for systematic biopsy. ${ }^{11}$ Conversely, micro-ultrasound is a relatively new imaging platform enabling high resolution ultrasound which operates at $29 \mathrm{MHz}$ compared to $9-12 \mathrm{MHz}$ for conventional urological ultrasound. This enables real-time spatial resolution of 70 microns, a 300\% improvement over conventional ultrasound resolution. Micro-ultrasound also has PRI-MUSTM (prostate risk identification using micro-ultrasound), an accompanying evidence-based analysis protocol which was first published last year. ${ }^{14}$ While mpMRI is performed ahead of time and the resulting report or imaging data is then used to target biopsies under real-time ultrasound guidance ("mpMRI/Ultrasound 
Fusion”) ${ }^{11}$, micro-ultrasound maintains the existing standard-of-care urological workflow and uses high resolution ultrasound to guide biopsies in real time. Micro-ultrasound, however provides significantly improved imaging resolution over conventional ultrasound imaging thereby enabling targeting of these biopsies. This study aims to provide an early feasibility comparison of these techniques, to determine whether their sensitives are close enough to merit a broader study. This study will also provide preliminary evidence for the combined use of these modalities for mpMRI/micro-ultrasound cognitive fusion.

\section{Methods}

\section{Subject selection}

Subjects currently enrolled in the Active Surveillance program at Urology of Virginia who met the inclusion and exclusion criteria (Table 1) were offered participation in the study. All procedures were approved by the local Institutional Review Board. This study is registered with ClinicalTrials.gov, NCT03035487. 9 subjects meeting these criteria were enrolled in December 2016, with all study procedures completed within the same month. All subjects provided informed consent prior to any study procedures, and all subjects successfully completed all planned study procedures. No adverse events were reported. Relevant clinical statistics on each subject are shown in Table 2.

\section{Multiparametric MRI}

Multi-parametric MRI (mpMRI) was performed on each patient up to 4 weeks prior to biopsy.

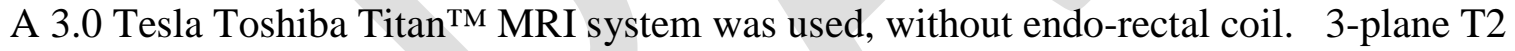
imaging, Diffusion weighted imaging (b-value 2,000) and Dynamic contrast enhanced sequences were used according to the PI-RADS v2 protocol. ${ }^{7}$ Resulting studies were read by a radiologist with 30 years of MRI experience and fellowship training in body imaging, who recently trained in PI-RADS v2 reporting. PI-RADS v2 scores from 3 (equivocal) to 5 (most suspicious) were marked as biopsy targets.

Despite his expertise in MRI, our primary reader was less experienced in prostate imaging ( $<1$ year). To ensure our results were applicable to both novice and expert prostate radiologists, the same mpMRI sequences were re-analyzed by a second PI-RADS expert reader with 5 years of experience in prostate MRI who has read over 1,000 cases.

The urologist was blinded to the mpMRI reports until the appropriate time in the biopsy procedure.

\section{Confirmatory/surveillance biopsy procedure}

The biopsy procedure consisted of imaging with conventional ultrasound to identify suspicious areas for targeting, followed by imaging with micro-ultrasound. Micro-ultrasound was used to identify suspicious areas and grade tissue according to the PRI-MUS protocol, as well as for realtime guidance of targeted and systematic biopsy samples. See Figure 1 for a detailed flow chart 
demonstrating the procedure. All biopsies were performed in the outpatient setting using the transrectal approach with subjects in the left lateral decubitus position, as is standard of care at our institution. Standard antibiotic prophylaxis and anti-coagulant management protocols were used, along with peri-prostatic nerve block anesthesia at the seminal vesicle junction.

\section{Conventional ultrasound imaging}

Conventional ultrasound imaging was performed by an operator with 26 years of urological ultrasound experience. The ExactVu ${ }^{\mathrm{TM}}$ system with an EV9C end-fire transducer ( $9 \mathrm{MHz}$, Exact Imaging, Markham, Canada) was used and hypoechoic areas and gross deviations in prostate margin were prospectively identified as suspicious. ${ }^{15}$ Images of each suspicious target and video loops sweeping through the gland were recorded for subsequent analysis.

\section{Micro-ultrasound imaging}

Micro-Ultrasound imaging was performed using the high resolution ExactVu ${ }^{\mathrm{TM}}$ system with an EV29L side-fire transducer (29 MHz, Exact Imaging, Markham, Canada). The operator had 3 years of experience using the micro-ultrasound modality. Risk of prostate cancer in each region was scored prospectively using the PRI-MUS protocol. ${ }^{14}$ Lesions exhibiting PRI-MUS 3, 4 and 5 features were specifically noted as biopsy targets. Images of each suspicious target and video loops sweeping through the gland were recorded for additional retrospective analysis. Only after all interrogation and scoring was complete were the MRI report and prior biopsy results unblinded. Micro-ultrasound was used to guide anesthesia delivery, measure the volume of the gland, and for real-time guidance of all systematic and targeted biopsy sampling.

Biopsy sampling with micro-ultrasound

Lesions identified on any of the three modalities were considered targets and acquired first with 2-3 samples per lesion, depending on size, via micro-ultrasound guided targeted biopsy. MRI and conventional ultrasound targets were acquired based on the radiology report and procedure notes, respectively, using cognitive fusion / real time visualization. When targets from multiple modalities overlapped, one set of targeted samples was taken, rather than a separate set for each modality from the same location. After all targeted samples were acquired, 12 standard systematic samples were taken with micro-ultrasound. In cases where a target position overlapped with a systematic position, the systematic sample was skipped to avoid unnecessary duplicated samples in any given region. All biopsy samples were taken transrectally, as is standard of care at our institution.

\section{Tracking MRI targeting accuracy}

As correct targeting of MRI lesions is not guaranteed during the cognitive fusion procedure, the inertial monitoring unit of the EV29L transducer (Exact Imaging Inc., Markham, Canada) was used to track transducer location. This unit provides angular information which is used to reconstruct the imaging plane of the transducer. With the scan plane identified, the lesion 
location can be compared based on distance to anatomical landmarks within the image, such as the rectal wall, apex and base capsule. A 3-dimensional positional error was calculated using the positional difference within the frame and the angular accuracy of the sensor.

\section{Statistical analysis}

McNemar's test with continuity correction implemented in MATLAB (Mathworks, Natick, USA) was used to assess the difference in sensitivity for pathology confirmed clinically significant targets between each pair of modalities. Clinically significant cancer was defined as any Gleason pattern 4 or 5 disease. PI-RADS 3 or higher lesions were considered targets for mpMRI, while PRI-MUS 3 or higher were considered targets on micro-ultrasound. Conventional ultrasound targets were hypoechoic regions or gross border deviations.

\section{Results}

Imaging comparison

High fidelity micro-ultrasound images were obtained on all subjects, allowing for volume measurement, anesthesia delivery and biopsy guidance in addition to PRI-MUS scoring of both suspicious regions and tissue in each systematic biopsy region. Images showing the lesions identified on both mpMRI and micro-ultrasound are shown in Figure 2. Micro-ultrasound images without any associated mpMRI target are shown in Figure 3. Video loops showing a complete sweep through the prostate using micro-ultrasound are provided in the online supplement.

mpMRI by readers 1 and 2 identified 2 and 5 targets (respectively) which contained clinically significant cancer on pathological analysis (GS7+), while micro-ultrasound identified 8. Table 3 shows a comparison of clinically significant lesions detected or missed by each modality. All lesions identified on either MRI or micro-ultrasound but not the other were assessed retrospectively. All clinically significant mpMRI targets identified except 1 were prospectively visible with micro-ultrasound, the remaining target was visible retrospectively. Of the 4 lesions seen on micro-ultrasound but not mpMRI, 2 were retrospectively assigned a grade of PI-RADS 3, and 1 PI-RADS 2 . The $4^{\text {th }}$ lesion was entirely MRI-invisible.

Targeting accuracy

Targeting accuracy was calculated for all MRI targets with comparable micro-ultrasound target, but no significant cancer on pathology $(\mathrm{N}=5)$ to ensure the micro-ultrasound did not lead the urologist away from the MRI target. Mean accuracy to lesion center was $3.3 \mathrm{~mm}$ [range 1.98$5.96 \mathrm{~mm}$ ]. 2 of these errors were larger than $3 \mathrm{~mm}$, suggesting possible misses, the remaining 3 had a mean accuracy of $2.2 \mathrm{~mm}$. 


\section{Sensitivity and specificity}

Micro-ultrasound outperformed mpMRI in sensitivity over a range of thresholds with 56-89\% sensitivity to clinically significant disease, compared to $22 \%$ for MRI reader 1 ( $\mathrm{p}=0.04$ McNemar's test). mpMRI reader 2's sensitivity was much higher at 33-56\% for clinically significant disease, depending on threshold, and while this is still considerably lower than microultrasound, the result is not statistically significant $(\mathrm{p}=0.37)$. Conventional ultrasound scored the lowest at $11 \%$, significantly lower than micro-ultrasound with $\mathrm{p}=0.02$. All three modalities demonstrated excellent specificity of greater than $90 \%$. Full results are presented in Table 4, along with the number of targets sampled from each modality.

\section{Clinical results}

Overall re-biopsy results were similar to other reports, ${ }^{4-6}$ with 2 (22.2\%) subjects receiving negative results, 4 (44.4\%) subjects maintaining a highest Gleason score of 6, and 3 (33.3\%) subjects upgraded to clinically significant disease based on at least one Gleason 7 or above sample. Among the 4 subjects who maintained Gleason 6 disease, number of positive cores and total percentage of cancer increased in 2 .

Targeted samples alone on both micro-ultrasound and MRI would have been sufficient to find all of the subjects upgraded to significant disease (3 of 3), conventional ultrasound however would only have found 1 of 3 . Total number of biopsy cores taken per subject averaged 17.2 (range 15-20).

\section{Discussion}

Both the sensitivity and upgrading results are consistent in suggesting that mpMRI using PIRADS, and now micro-ultrasound using PRI-MUS provide an improved ability to detect significant prostate cancer compared to conventional ultrasound. Despite the small sample size and feasibility nature of the study, statistical significance was also reached comparing microultrasound sensitivity against the novice PI-RADS mpMRI reader.

Conclusions from this study are limited by the small number of subjects, single institution, and single- micro-ultrasound reader. As such, we are unable to draw any conclusions on inter-reader variability. Inter-reader variability is an important metric for any diagnostic tool, and has been studied for PRI-MUS and at length for PI-RADS. ${ }^{11,14,16,17}$ Additionally, this study made use of cognitive fusion on the micro-ultrasound platform, rather than a MRI/US fusion device. Though current evidence on the differences between these techniques is equivocal, ${ }^{18}$ some studies suggest that using an MRI/US fusion device may have improved the accuracy of MRI targeting.

The use of cognitive fusion introduces the risk of bias in cases where both an mPMRI and micro-ultrasound lesion is noted in the same region. During the biopsy procedure, these lesions could have been falsely considered equivalent. This would have led to the micro-ultrasound lesion being sampled rather than the mpMRI lesion. Since micro-ultrasound demonstrated 
higher sensitivity than mpMRI this is less of a concern, however there were still 5 cases where both micro-ultrasound and MRI were positive with a non-significant cancer biopsy result. If the micro-ultrasound lesion misled the operator, it is possible these lesions could have yielded significant results. However, it is equally possible that any of the 4 significant MRI lesions were significant only because of the observed micro-ultrasound lesion. As described above, to mitigate this bias we have measured the sampling accuracy and determined that our targeting was generally accurate with 2 possible misses. Future studies involving a more accurate fusion system may be necessary.

Due to the population studied, it was not possible to correlate findings with whole mount radical prostatectomy specimens. This limits the analysis in that there may be other areas of prostate tissue positive for clinically significant cancer which were not discovered by biopsy. This is particularly relevant to the PPV and Specificity results because targets with benign biopsy results may have found significant disease on radical prostatectomy. Further, since other areas of cancer may have been present, all NPV and sensitivity results must be considered relative to the other modalities tested rather than absolute.

Despite these limitations, using only targeted samples micro-ultrasound correctly diagnosed all 3 upgraded subjects. Unfortunately, the small number of subjects do not allow us to draw any conclusions on whether it may be possible to recommend only targeted biopsy in the future. Future studies with larger enrollment numbers, preferably across multiple sites, will be needed.

The current version of the PRI-MUS protocol used in this study is limited in only providing guidance on suspicious regions of the peripheral zone. While $70-80 \%$ of prostate cancers involve the peripheral zone, ${ }^{15,19}$ targeting using PRI-MUS alone may miss cancer when it is isolated to the anterior zone. Despite the well-known phenomenon of ultrasound signal loss in the deeper anterior zone, image quality was good for all cases performed in this study. Microultrasound could (in 2 cases retrospectively) visualize all mpMRI targets except for 1 anterior target. This supports the use of cognitive fusion both in this study, and in general when a microultrasound system is used. The high quality of anterior zone imaging (for example see Figure 3d) in this study holds promise for the next version of the PRI-MUS protocol to provide guidance on transition and anterior zone disease.

Our results demonstrate lower sensitivity than has been reported previously for mpMRI at some centers. ${ }^{20,21}$ The cause for this discrepancy is unclear, though may include the low number of subjects, low-grade disease which is more difficult to see on mpMRI, and the known variability of mpMRI between readers and sites. ${ }^{16,22}$ Further, recent work from a larger active surveillance cohort biopsied using the UroNav fusion system (Invivo) found a similar sensitivity, ${ }^{23}$ suggesting that this patient population may be more challenging to mpMRI. Finally, the lower sensitivity may also be caused by the improved targeting of micro-ultrasound. Finding more cancer in another modality would increase the total number of positive biopsy 
samples and therefore decrease the sensitivity of all other modalities, in this case raising mpMRI sensitivity to $100 \%$ if micro-ultrasound targets are removed. The large difference between novice and expert mpMRI readers was consistent with reports in the literature documenting similar variability for staging. ${ }^{11,17,24-26}$

\section{Conclusion}

Micro-ultrasound is a new modality, just beginning to build evidence and expertise around its use, however preliminary work such as this demonstrates that it can successfully visualize and target biopsies for prostate cancer. Maintaining the existing urological workflow of ultrasound guided biopsies simplifies both the patient experience and the clinical effort required for cancer detection (single-specialist, single office visit, no reporting or fusion requirements). Although this study was small, it suggests that micro-ultrasound may be comparable to mpMRI in ways that conventional ultrasound is not. Thus, we conclude that the technology is a promising tool for detecting and targeting prostate cancer in the Active Surveillance population, and larger multi-site and multi-reader studies are warranted. 


\section{References}

1. Ferlay J, Parkin DM, Steliarova-Foucher E. Estimates of cancer incidence and mortality in Europe in 2008. Eur J Cancer 2010;46:765-81.

2. Bray F, Ren JS, Masuyer E, et al. Global estimates of cancer prevalence for 27 sites in the adult population in 2008. Int J Cancer 2013;132:1133-45.

3. American Cancer Society. Cancer facts \& figures 2013. Am Cancer Soc 2013;2010.

4. Jain S, Loblaw A, Vesprini D, et al. Gleason upgrading with time in a large prostate cancer active surveillance cohort. J Urol 2015;194:79-84.

5. Al Otaibi M, Ross P, Fahmy N, et al. Role of repeated biopsy of the prostate in predicting disease progression in patients with prostate cancer on active surveillance. Cancer 2008;113:286-92.

6. Berglund RK, Masterson TA, Vora KC, et al. Pathologic upgrading and upstaging with immediate repeat biopsy for patients eligible for active surveillance. $J$ Urol 2008;180:1964-8.

7. Weinreb JC, Barentsz JO, Choyke PL, et al. PI-RADS prostate imaging - reporting and data system: 2015, version 2. Eur Urol 2016;69:16-40.

8. Barentsz JO, Weinreb JC, Verma S, et al. Synopsis of the PI-RADS v2 guidelines for multiparametric prostate magnetic resonance imaging and recommendations for use. Eur Urol 2016;69:41-9.

9. Pavlovich CP, Cornish TC, Mullins JK, et al. High-resolution transrectal ultrasound: Pilot study of a novel technique for imaging clinically localized prostate cancer. Urol Oncol 2013:1-6.

10. Valerio M, Donaldson I, Emberton M, et al. Detection of clinically significant prostate cancer using magnetic resonance imaging-ultrasound fusion targeted biopsy: A systematic review. Eur Urol 2015;68:8-19.

11. Taneja SS, Gaitonde K, Eggener SE, et al. Prostate MRI and MRI-targeted biopsy in patients with prior negative biopsy. Am Urol Assoc 2016:1-19.

12. Rosenkrantz AB, Ginocchio LA, Cornfeld D, et al. Interobserver reproducibility of the PI-RADS version 2 lexicon: A multicenter study of six experienced prostate radiologists. Radiology 2016;280:793-804.

13. Mertan F V., Greer MD, Shih JH, et al. Prospective evaluation of the prostate imaging reporting and data system version 2 (PI-RADSv2) for prostate cancer detection. $J$ Urol 2016;196:690-6.

14. Ghai S, Eure G, Fradet V, et al. Assessing cancer risk on novel $29 \mathrm{MHz}$ micro-ultrasound images of the prostate: Creation of the micro-ultrasound protocol for prostate risk identification. J Urol 2016;196:562-9.

15. Toi A. The Prostate. Rumack CM, Wilson SR, Charboneau JW, et al., eds. Diagnostic Ultrasound, $4^{\text {th }}$ ed 2011:392-428.

16. Vargas $\mathrm{H}$ a, Akin O, Afaq A, et al. Magnetic resonance imaging for predicting prostate biopsy findings in patients considered for active surveillance of clinically low risk prostate cancer. J Urol 2012;188:1732-8.

17. Rosenkrantz AB, Lim RP, Haghighi M, et al. Comparison of interreader reproducibility 
of the prostate imaging reporting and data system and likert scales for evaluation of multiparametric prostate MRI. Am J Roentgenol 2013;201:612-8.

18. Wegelin O, van Melick HHE, Hooft L, et al. Comparing three different techniques for magnetic resonance imaging-targeted prostate biopsies: A systematic review of in-bore versus magnetic resonance imaging-transrectal ultrasound fusion versus cognitive registration. Is there a preferred technique? Eur Urol 2017;71:517-31.

19. Sánchez-Chapado M, Olmedilla G, Cabeza M, et al. Prevalence of prostate cancer and prostatic intraepithelial neoplasia in Caucasian Mediterranean males: An autopsy study. Prostate 2003;54:238-47.

20. Ahmed HU, El-Shater Bosaily A, Brown LC, et al. Diagnostic accuracy of multiparametric MRI and TRUS biopsy in prostate cancer (PROMIS): A paired validating confirmatory study. Lancet 2017;389:815-22.

21. Schouten MG, van der Leest M, Pokorny M, et al. Why and where do we miss significant prostate cancer with multi-parametric magnetic resonance imaging followed by magnetic resonance-guided and transrectal ultrasound-guided biopsy in biopsy-naïve men? Eur Urol 2017;71:896-903.

22. Branger $\mathrm{N}$, Maubon $\mathrm{T}$, Traumann $\mathrm{M}$, et al. Is negative multiparametric magnetic resonance imaging really able to exclude significant prostate cancer? The real-life experience. BJU Int 2017;119:449-55.

23. Ma TM, Tosoian JJ, Schaeffer EM, et al. The role of multiparametric magnetic resonance imaging/ultrasound fusion biopsy in active surveillance. Eur Urol 2017;71:174-80.

24. Fütterer JJ, Heijmink SWTPJ, Scheenen TWJ, et al. Prostate cancer: Local staging at 3-T endorectal MR imaging - early experience. Radiology 2006;238:184-91.

25. Seltzer SE, Getty DJ, Tempany CM, et al. Staging prostate cancer with MR imaging: a combined radiologist-computer system. Radiology 1997;202:219-26.

26. Latchamsetty KC, Borden LS, Porter CR, et al. Experience improves staging accuracy of endorectal magnetic resonance imaging in prostate cancer: What is the learning curve? Can J Urol 2007;14:3429-34. 


\section{Figures and Tables}

Fig. 1. Study procedure. A multiparametric magnetic resonance imaging (mpMRI) was performed up to four weeks prior to the biopsy session. During the biopsy session, imaging was first performed with conventional transrectal ultrasound, identifying suspicious regions to target. The conventional ultrasound transducer was then removed from the rectum and the microultrasound transducer inserted. The prostate was interrogated to identify prostate risk identification for micro-ultrasound (PRI-MUS) 3, 4, and 5 areas for targeting. After all target lesions were identified, anesthesia was delivered and the mpMRI report was unblinded. Targeted samples were then taken from targets seen on any modality, along with up to 12 systematic samples.

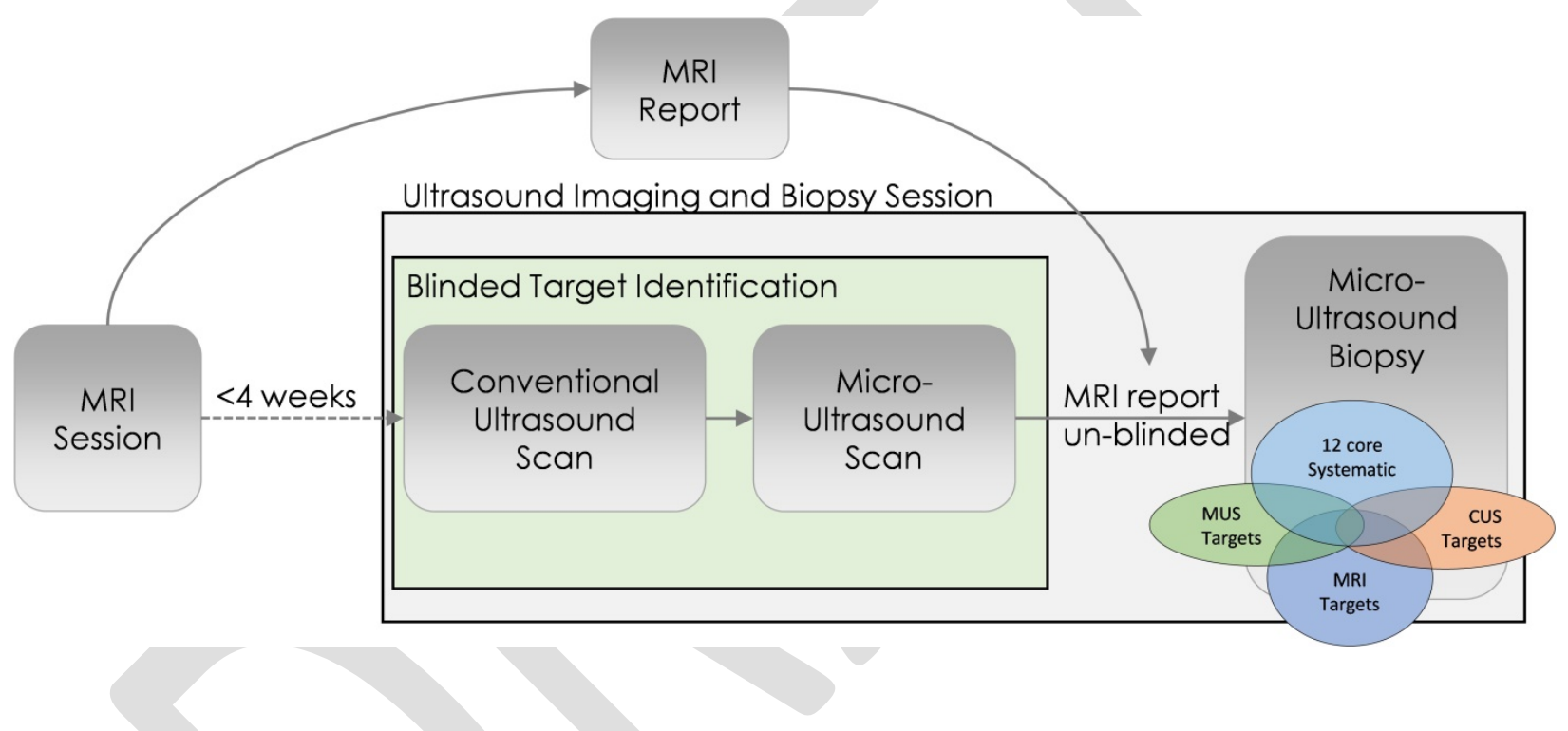

Fig. 2. Lesions prospectively identified on both multiparametric magnetic resonance (mpMRI) and micro-ultrasound. The upper grouping $(\boldsymbol{A}-\boldsymbol{D})$ shows a single lesion, which was visible in right mid-apex peripheral zone of subject 3. The MRI is shown in panels $\boldsymbol{A}$ (DWI), and $\boldsymbol{B}$ (axial T2-wieghted), along with a locator drawing showing the position of the slice, and a blue circle highlighting the lesion. Panel $\boldsymbol{D}$ shows the same data resliced to match the para-sagittal view of the micro-ultrasound, along with the same locator and circle highlighting the lesion. Panel $\boldsymbol{C}$ shows the micro-ultrasound image, where the lesion is quite obvious. This lesion was labeled as a prostate imaging reporting and data system (PI-RADS) 4 on mpMRI and a prostate risk identification for micro-ultrasound (PRI-MUS) 5 (mixed echo lesion) on micro-ultrasound. Pathology determined the lesion to be Gleason 9 with $20 \%$ core length $(3 \mathrm{~mm}$ ). The second grouping $(\boldsymbol{E}-\boldsymbol{G})$ shows a single lesion from the left base-mid peripheral zone of subject 5 . The image panels are as described above, T2-weighted MRI in $\boldsymbol{F}$, DWI in $\boldsymbol{E}$, resliced MRI in $\boldsymbol{I}$, 
conventional ultrasound in $\boldsymbol{H}$, and micro-ultrasound frame in $\boldsymbol{G}$. This lesion was labeled a PIRADS 4 on mpMRI, and a PRI-MUS 5 on micro-ultrasound, pathology determined this lesion to be a Gleason 7 with $10 \%$ core length $(1.3 \mathrm{~mm})$. The micro-ultrasound is interesting in this case because it shows a PRI-MUS 5 centre (bulging capsule) and larger PRI-MUS 4 surround (smudgy texture). The lower two groupings show lesions which were identified by reader 2, but not by reader 1 . These lesions were identified as a PI-RADS 5/PRI-MUS 5 (bulging capsule) lesion found to contain 10\% Gleason score (GS) 8 (J-M), and a PI-RADS 5/PRI-MUS 5 (bulging capsule) lesion found to contain 90\% GS 7 (N-R).
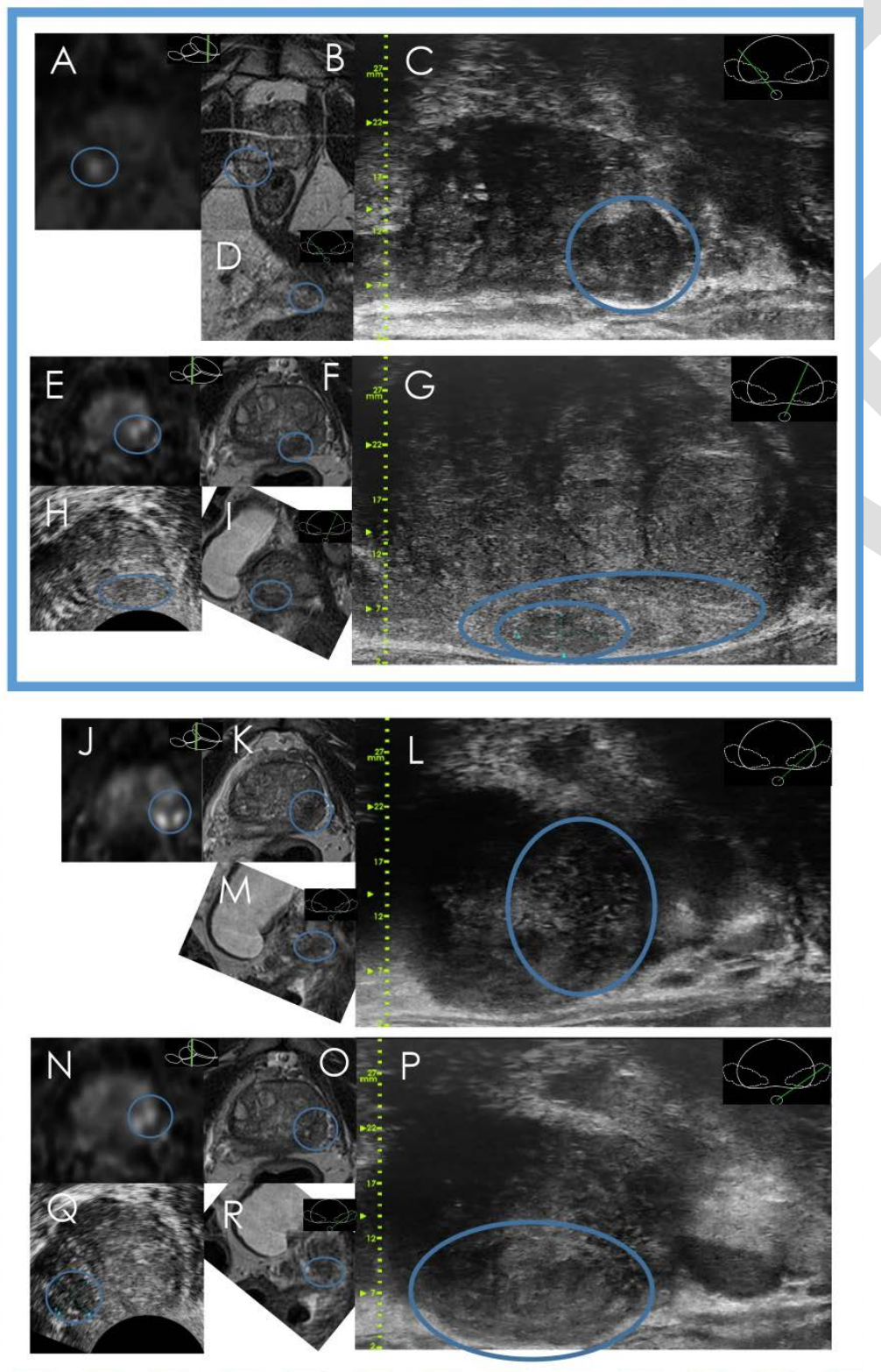
Fig. 3. Lesions prospectively identified on micro-ultrasound only. Micro-ultrasound frames showing lesions with pathology confirmed clinically significant cancer. Prostate risk identification for micro-ultrasound (PRI-MUS) lesions are circled in blue. (A) PRI-MUS 3 (mild heterogeneity) lesion found to contain 5\% Gleason score (GS) 7; (B) PRI-MUS 3 (mild heterogeneity) lesion found to contain 10\% GS 7; (C) PRI-MUS 3 (mild heterogeneity) found to contain 5\% GS 7; (D) PRI-MUS 5 (mixed echo lesion) found to contain 5\% GS 7.
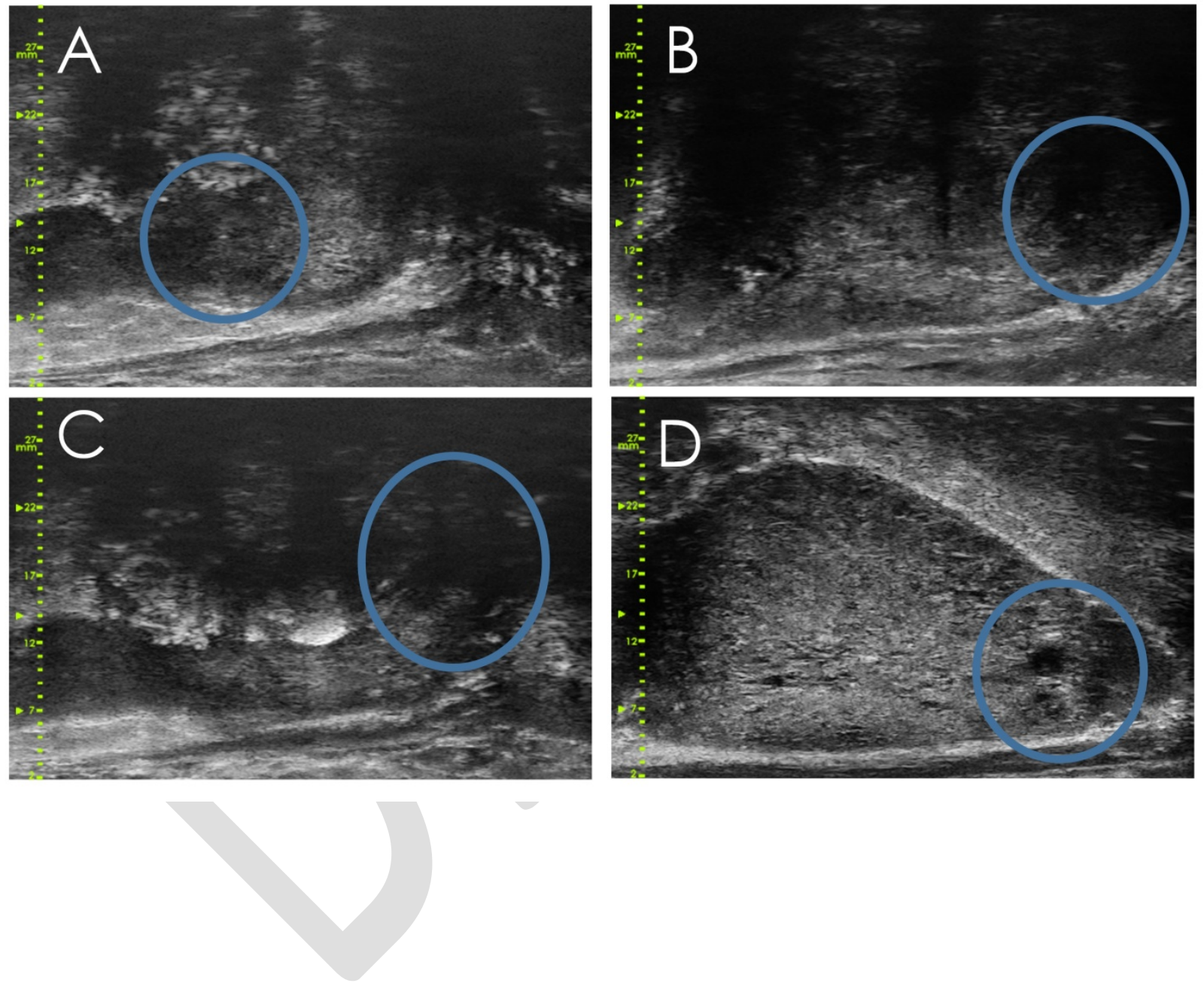


\begin{tabular}{|c|c|}
\hline Inclusion criteria & Exclusion criteria \\
\hline $\begin{array}{ll}- & \text { Men } \geq 40 \text { and }<80 \text { years of age } \\
- & \text { History of prostate cancer } \\
- & \text { Indication for prostate biopsy under } \\
& \text { existing active surveillance protocol } \\
& \text { (confirmatory or surveillance) } \\
- & \text { PSA }<20 \text { ng/mL } \\
- & \text { Clinical stage }<\text { cT2c }\end{array}$ & $\begin{array}{ll}- & \text { Undergoing TRUS-guided prostate } \\
\text { biopsy in the OR under anesthesia } \\
-\quad \text { Known prostate volume (from prior } \\
\text { imaging) of }>60 \text { cc } \\
-\quad \text { Anorectal abnormalities preventing } \\
\text { TRUS-guided prostate biopsy } \\
-\quad \text { Unable to provide informed consent } \\
-\quad \text { Contraindication to MRI or gadolinium } \\
\text { chelate contrast }\end{array}$ \\
\hline
\end{tabular}

MRI: magnetic resonance imaging; OR: operating room; PSA: prostate-specific antigen; TRUS: transrectal ultrasound.

Table 2. Summary of subject statistics on enrollment

\begin{tabular}{|c|c|c|c|c|c|c|c|c|}
\hline \multirow[b]{2}{*}{ Subject } & \multirow[b]{2}{*}{ Age } & \multirow[b]{2}{*}{$\begin{array}{l}\text { Vol } \\
\text { (cc) }\end{array}$} & \multirow[b]{2}{*}{$\begin{array}{c}\text { PSA } \\
(\mathrm{ng} / \mathrm{mL})\end{array}$} & \multirow[b]{2}{*}{ DRE } & \multirow[b]{2}{*}{$\begin{array}{c}\text { Years since } \\
\text { diagnosis (\# } \\
\text { biopsies) }\end{array}$} & \multicolumn{3}{|c|}{ Most recent biopsy results } \\
\hline & & & & & & $\begin{array}{c}\text { Fraction } \\
\text { positive } \\
\text { cores }\end{array}$ & $\begin{array}{c}\text { Highest } \\
\text { grade }\end{array}$ & $\begin{array}{c}\text { Highest } \\
\text { percentage }\end{array}$ \\
\hline EI-01 & 73 & 33 & 7.75 & cT1c & $3(2)$ & $1 / 12$ & $3+3=6$ & 3 \\
\hline EI-02 & 65 & 37 & 4.99 & cT1c & $2(1)$ & $4 / 12$ & $3+3=6$ & 50 \\
\hline EI-03 & 62 & 40 & 4.71 & cT2a & $<1(1)$ & $1 / 12$ & $3+3=6$ & 30 \\
\hline EI-04 & 75 & 44 & 7.86 & cT1c & $1(1)$ & $2 / 12$ & $3+3=6$ & 10 \\
\hline EI-05 & 68 & 51 & 5.90 & cT2a & $9(2)$ & $1 / 8$ & $3+3=6$ & 5 \\
\hline EI-06 & 66 & 53 & 5.36 & cT1c & $3(2)$ & $2 / 12$ & $3+3=6$ & 5 \\
\hline EI-07 & 60 & 29 & 4.85 & cT1c & $1(1)$ & $1 / 12$ & $3+3=6$ & 5 \\
\hline EI-08 & 54 & 25 & 6.40 & cT1c & $1(1)$ & $1 / 12$ & $3+3=6$ & 47 \\
\hline EI-09 & 67 & 37 & 6.42 & cT1c & $<1(1)$ & $5 / 12$ & $3+3=6$ & 10 \\
\hline
\end{tabular}

DRE: digital rectal exam; PSA: prostate-specific antigen. 


\begin{tabular}{|c|c|c|c|c|}
\hline & $\begin{array}{c}\text { Positive } \\
\text { micro- } \\
\text { ultrasound } \\
\end{array}$ & $\begin{array}{c}\text { Negative } \\
\text { micro- } \\
\text { ultrasound }\end{array}$ & $\begin{array}{c}\text { Positive } \\
\text { conventional } \\
\text { ultrasound } \\
\end{array}$ & $\begin{array}{c}\text { Negative } \\
\text { conventional } \\
\text { ultrasound }\end{array}$ \\
\hline $\begin{array}{l}\text { Positive mpMRI } \\
\text { (reader 1) }\end{array}$ & 2 & 0 & 0 & 2 \\
\hline $\begin{array}{l}\text { Negative mpMRI } \\
\text { (Reader 1) }\end{array}$ & 6 & 1 & 1 & 6 \\
\hline $\begin{array}{l}\text { Positive mpMRI } \\
\text { (reader 2) }\end{array}$ & 4 & 1 & 1 & 3 \\
\hline $\begin{array}{l}\text { Negative mpMRI } \\
\text { (reader 2) }\end{array}$ & 4 & 0 & 0 & 5 \\
\hline $\begin{array}{l}\text { Positive conventional } \\
\text { ultrasound }\end{array}$ & 1 & 0 & & \\
\hline $\begin{array}{l}\text { Negative conventional } \\
\text { ultrasound }\end{array}$ & 7 & 1 & & \\
\hline
\end{tabular}

mpMRI: multiparametric magnetic resonance imaging; PI-RADS: prostate imaging reporting and data system; PRI-MUS: prostate risk identification for micro-ultrasound.

\begin{tabular}{|l|c|c|c|c|c|c|}
\hline Table 4. Per-lesion sample statistics (prospective data only) & \multicolumn{3}{l|}{} \\
\hline Modality & $\begin{array}{c}\text { Number of } \\
\text { targets }\end{array}$ & $\begin{array}{c}\text { Number } \\
\text { positive }\end{array}$ & Sensitivity & Specificity & PPV & NPV \\
\hline $\begin{array}{l}\text { Conventional } \\
\text { ultrasound }\end{array}$ & 9 & 1 & $11 \%$ & $93 \%$ & $11 \%$ & $93 \%$ \\
\hline $\begin{array}{l}\text { mpMRI (reader 1) } \\
\text { PI-RADS 3+ }\end{array}$ & 6 & 2 & $22 \%$ & $96 \%$ & $33 \%$ & $94 \%$ \\
\hline $\begin{array}{l}\text { mpMRI (reader 2) } \\
\text { PI-RADS 3+ }\end{array}$ & 20 & 5 & $56 \%$ & $86 \%$ & $25 \%$ & $96 \%$ \\
\hline $\begin{array}{l}\text { mpMRI (reader 2) } \\
\text { PI-RADS 5 only }\end{array}$ & 6 & 3 & $33 \%$ & $97 \%$ & $50 \%$ & $95 \%$ \\
\hline $\begin{array}{l}\text { Micro-ultrasound } \\
\text { PRI-MUS 3+ }\end{array}$ & $67^{*}$ & 8 & $89 \%$ & $45 \%$ & $12 \%$ & $98 \%$ \\
\hline $\begin{array}{l}\text { Micro-ultrasound } \\
\text { PRI-MUS 5 only }\end{array}$ & 14 & 5 & $56 \%$ & $92 \%$ & $36 \%$ & $96 \%$ \\
\hline Systematic only & 41 & 0 & & & & \\
\hline
\end{tabular}

*Only a single biopsy core was taken from PRI-MUS 3 targets, while 2-3 cores were taken from PRI-MUS 4 and 5, and PI-RADS 3-5 lesions. mpMRI: multiparametric magnetic resonance imaging; NPV: negative predictive value; PI-RADS: prostate imaging; reporting and data system; PPV: positive predictive value PRI-MUS: prostate risk identification for microultrasound. 


\begin{tabular}{|c|c|c|c|c|c|c|c|c|c|}
\hline \multirow{2}{*}{ Subject } & \multirow{2}{*}{$\begin{array}{c}\text { PSA } \\
\text { velocity } \\
\text { (ng/ml/yr) }\end{array}$} & \multicolumn{4}{|c|}{$\begin{array}{c}\text { Modalities targeting highest GS lesion } \\
\text { (other lesion) }\end{array}$} & \multicolumn{2}{|c|}{ Previous biopsy } & \multicolumn{2}{|c|}{ Current biopsy } \\
\hline & & US & $\begin{array}{l}\text { Micro- } \\
\text { US }\end{array}$ & $\begin{array}{c}\text { mpMRI } \\
1\end{array}$ & $\begin{array}{l}\text { mpMRI } \\
2\end{array}$ & $\begin{array}{c}\text { Max } \\
\text { GS }\end{array}$ & $\begin{array}{l}\text { Positive } \\
\text { cores }\end{array}$ & $\begin{array}{c}\text { Max } \\
\text { GS }\end{array}$ & $\begin{array}{c}\text { Positive } \\
\text { cores }\end{array}$ \\
\hline EI-01 & 1.76 & & & & & $3+3=6$ & $1 / 12$ & N/A & $0 / 16$ \\
\hline EI-02 & -0.48 & & $\checkmark$ & & $\checkmark$ & $3+3=6$ & $4 / 12$ & $3+3=6$ & $6 / 19$ \\
\hline EI-03 & $-1.08^{*}$ & & $\checkmark$ & $\checkmark$ & $\checkmark$ & $3+3=6$ & $1 / 12$ & $4+5=9$ & $9 / 18$ \\
\hline EI-04 & 1.68 & & $\checkmark$ & & $\sqrt{ }$ & $3+3=6$ & $2 / 12$ & $3+3=6$ & $3 / 15$ \\
\hline EI-05 & $0.05^{*}$ & (GS7) & $\checkmark$ & (GS7) & $\checkmark$ & $3+3=6$ & $1 / 8$ & $4+4=8$ & $6 / 16$ \\
\hline EI-06 & 0.94 & & $\checkmark$ & (GS6) & $\checkmark$ & $3+3=6$ & $2 / 12$ & $3+4=7$ & $3 / 18$ \\
\hline EI-07 & 0.04 & & & & & $3+3=6$ & $1 / 12$ & N/A & $0 / 16$ \\
\hline EI-08 & 1.31 & $\checkmark$ & $\checkmark$ & $\checkmark$ & $\checkmark$ & $3+3=6$ & $1 / 12$ & $3+3=6$ & $4 / 17$ \\
\hline EI-09 & 1.36 & & $\checkmark$ & 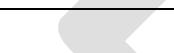 & $\checkmark$ & $3+3=6$ & $5 / 12$ & $3+3=6$ & $7 / 20$ \\
\hline
\end{tabular}

Highest Gleason score (GS) biopsy sample and number of positive samples are shown for each subject before and after the current biopsy procedure. The modalities that successfully targeted at least one of the highest GS samples are marked with a checkmark. If a modality failed to target the highest GS sample, the highest GS targeted is shown in parentheses. ${ }^{*}$ PSA drawn on 5-alpha reductase inhibitor. GS: Gleason score; mpMRI: multiparametric magnetic resonance imaging; PSA: prostate-specific antigen; US: ultrasound. 